\title{
HERA-signature based searches I: events with isolated leptons and missing transverse momentum at HERA
}

\author{
James Ferrando \\ University of Oxford - Dept of Physics \\ Denys Wilkinson Building Keble Road, Oxford OX1 3RH - United Kingdom
}

\begin{abstract}
Recent results of searches for events containing isolated leptons and missing transverse momentum at HERA are presented. Searches in this final state have yielded notable excesses over Standard Model expectations in the past. Searches for isolated leptons in channels corresponding to all three generations of leptons over the full HERA running period are now available. The combined H1+ZEUS results for searches for electrons and muons are compatible with the Standard Model.
\end{abstract}

\section{Introduction}

Searches for events containing isolated leptons and large missing transverse momentum have long been considered to be a high priority at HERA. The dominant genuine source of such events within the Standard Model (SM) is real $W$-boson production, with subsequent leptonic decay, there is also a small expected event rate from real $Z$-boson production with subsequent decay to neutrinos. The total expected cross section for these heavy boson production processes at HERA is of the order of $1 \mathrm{pb}$ at leading order [1]. Quantum chromodynamics (QCD) corrections to this cross-section at next-to-leading-order (NLO) have been calculated [2]: the overall magnitude of the cross section is not changed; the uncertainty on the expected $W$-production cross section after including these corrections is approximately $15 \%$. Mismeasurement of events produced by other SM processes such as neutral current (NC) deep inelastic scattering (DIS), charged current (CC) DIS and dilepton production also produce measurable event rates matching this topology and such events must be removed with cuts; this class of events are referred to as 'fake signal'.

Events containing isolated leptons and large missing transverse momentum would be produced at HERA by several models of physics beyond the SM such as single-top-production via flavour-changing neutral currents [3] and $\mathcal{R}$-parity violating supersymmetry [4]. Both $\mathrm{SM}$ vector-boson production and these new physics models are expected to produce leptons with large values of transverse momentum $\left(P_{T}^{l}\right)$. However, the new physics models would be expected to produce events with large values of hadronic transverse momentum $\left(P_{T}^{X}\right)$ whereas SM vector boson production is expected to predominantly produce events with small $P_{T}^{X}$. The low expected production rate of these events from the SM and the clean topology make this an ideal environment in which to search for evidence for these new physics models.

Previous searches for isolated electrons and muons by the $\mathrm{H} 1$ collaboration using data taken during the 1994-2000 (HERA-I) running period [5, 6] yielded an excess of events over the SM predictions at large values $(>25 \mathrm{GeV})$ of $P_{T}^{X}$. Searches for isolated-electron events in the HERA-I data by the ZEUS collaboration in the context of single- $W$ and single top production $[7,8]$ did not confirm this excess. Searches for isolated-tau events in the HERA-I data were also performed by both H1[9] and ZEUS[10], the results were compatible with SM predictions. With a four-fold increase in integrated luminosity relative to HERA-I, the

DIS 2008 
2003-2007 (HERA-II) running period offers data that can resolve the apparent discrepancy between the previous results of the H1 and ZEUS collaborations.

In these proceedings the latest results from searches for isolated-electron and isolatedmuon events at $\mathrm{H} 1$ and ZEUS are shown. The result of combining these H1 and ZEUS searches is presented. Finally an H1 search for isolated tau leptons is presented. These new results include data collected during the HERA-II running period. Results that interpret searches for this class of events in the context of measurements of single- $W$ production or single-top production are discussed elsewhere [11].

\section{Searches for isolated electrons or muons}

Searches for events containing isolated leptons (electrons or muons) and large missing transverse momentum have recently been performed at HERA by both the H1 [12] and ZEUS [13] collaborations. The main source of fake signal in the electron search are CC DIS at low values of $P_{T}^{X}$ and NC DIS at high $P_{T}^{X}$. In the muon search the main source of fake signal is Bethe-Heitler dimuon production. In both searches the fraction of the SM prediction arising from $W$ production is typically over $60 \%$

The H1 search is performed in the same kinematic region as was explored in the previous HERAI search [6]. This region is defined by $P_{T}^{l}>10 \mathrm{GeV}$ and the polar angle of the lepton, $\theta_{l}$, lying in the range $5<\theta_{l}<140^{\circ}$. The kinematic region in the muon phase space is additionally limited to $P_{T}^{X}>12 \mathrm{GeV}$. The data set considered corresponds to an integrated luminosity of $294 \mathrm{pb}^{-1}\left(184 \mathrm{pb}^{-1}\right)$ for $e^{+} p\left(e^{-} p\right)$ collisions.

The distribution of $P_{T}^{X}$ is compared to SM Monte Carlo (MC) separately for $e^{+} p$ and $e^{-} p$ collision data in Fig. 1. It can be seen that in $e^{+} p$ collsions the data agree very well with the SM at small values of $P_{T}^{X}$ but is generally above the SM predictions at larger values of $P_{T}^{X}$. For $P_{T}^{X}>25 \mathrm{GeV}, 21$ (3) events are observed where $8.9 \pm 1.5(6.9 \pm 1.0)$ are expected for $e^{+} p\left(e^{-} p\right)$ collsions. The excess of data over $\mathrm{SM}$ in $e^{+} p$ collisions is present in both the
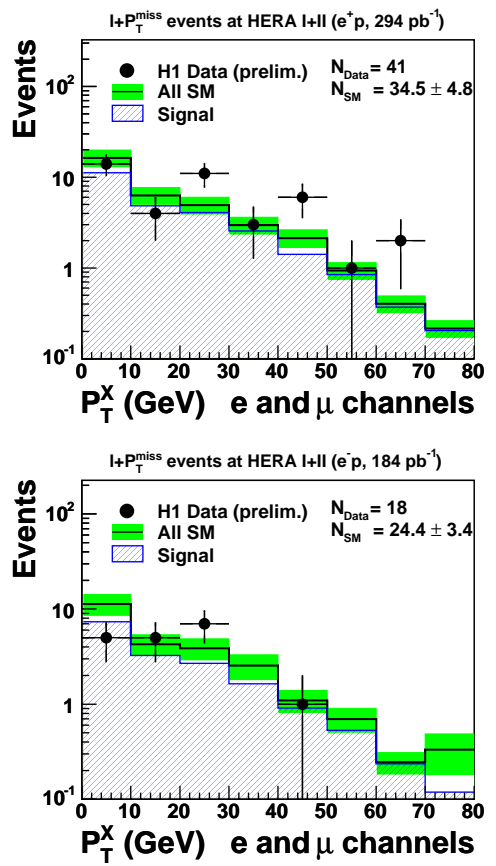

Figure 1: The distribution of $P_{T}^{X}$ for events selected in the H1 isolated lepton search for (top) $e+p$ collisions and (bottom) $e^{-} p$ collisions. electron and muon searches, where the numbers seen are respectively 11 and 10 compared to expectations of $4.7 \pm 0.9$ and $4.2 \pm 0.7$.

The ZEUS search is performed in a kinematic region defined by $P_{T}^{l}>10 \mathrm{GeV}$ and $15<\theta_{l}<120^{\circ}$. The kinematic region in the muon phase space is additionally limited to $P_{T}^{X}>12 \mathrm{GeV}$. The data set considered corresponds to an integrated luminosity of $286 \mathrm{pb}^{-1}\left(206 \mathrm{pb}^{-1}\right)$ for $e^{+} p\left(e^{-} p\right)$ collisions. The distribution of $P_{T}^{X}$ is compared to SM MC separately for $e^{+} p$ and $e^{-} p$ collision data in Fig. 2. It can be seen that the data agrees 
well with the MC at High $P_{T}^{X}$. A small deficit of data with respect to $\mathrm{MC}$ can be seen at low $P_{T}^{X}$ in $e^{+} p$ collisions, similar to that observed by the $\mathrm{H} 1$ collaboration in $e^{-} p$ collisions.
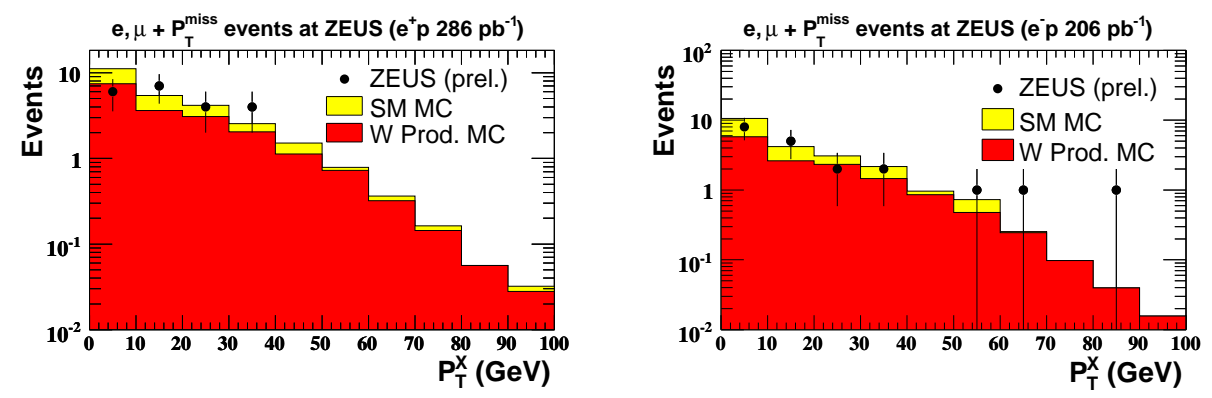

Figure 2: The distribution of $P_{T}^{X}$ for events selected in the ZEUS isolated-lepton search for (left) $e+p$ collisions and (right) $e^{-} p$ collisons.

\section{Combined H1+ZEUS search}

The H1 and ZEUS isolated-electron or -muon searches are both sensitive to the same physics processes, with similar efficiencies and fake rates. This enables the searches to be combined at the data level in the common kinematic region: $P_{T}^{l}>10 \mathrm{GeV}$ and $15<\theta_{l}<120^{\circ}$. The kinematic region in the muon phase space is additionally limited to $P_{T}^{X}>12 \mathrm{GeV}$. This combination has recently been performed [14] yielding a total integrated luminosity of $0.97 \mathrm{fb}^{-1}$.

The distribution of $P_{T}^{X}$ is compared to SM MC separately for $e^{+} p$ and $e^{-} p$ collision data in Fig. 3. The data agree beautifully with the SM predictions across the full $P_{T}^{X}$ range. For $P_{T}^{X}>25, \mathrm{GeV}, 29$ events are observed compared to an expectation of $25.3 \pm 3.2$. This combined result is compatible with SM $W$ production.

\section{Searches for isolated taus}

Searches for events containing isolated $\tau$ leptons and large missing transverse momentum at HERA are extremely challenging. The short lifetime of the $\tau$ means that it decays before it can be directly detected. Production of isolated taus which decay into leptons $\left(\tau^{-} \rightarrow e^{-} \bar{\nu}_{e} \nu_{\tau}, \mu^{-} \bar{\nu}_{\mu} \nu_{\tau}\right.$ and charge conjugates) are experimentally indistinguishable from direct production of isolated electrons or muons. The $\tau$ decay channel of choice for these searches at high transverse momentum is therefore the single-prong hadronic decay. Different algorithms are used to separate the highly collimated 
hadronic jets produced by isolated taus from jets produced by other sources. Charged current DIS events where $\tau$ candidates are found in the hadronic final state constitute the largest fake signal contribution in this search.

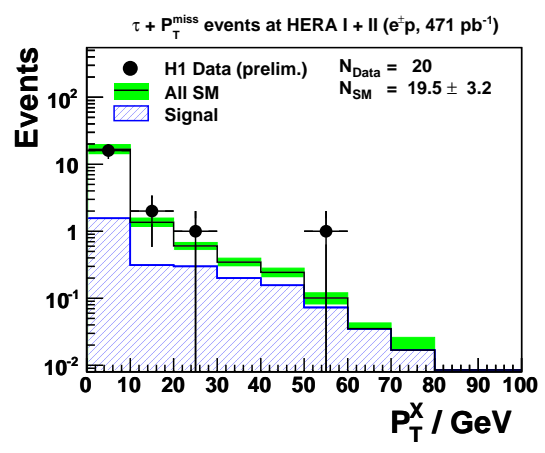

Figure 4: The distribution of $P_{T}^{X}$ for events selected in the $\mathrm{H} 1 \tau$ search.

The H1 collaboration has recently produced a new search for isolated $\tau$ leptons [15] using $287 \mathrm{pb}^{-1}\left(184 \mathrm{pb}^{-1}\right)$ data from $e^{+} p\left(e^{-} p\right)$ collisions in both HERA-I and HERA-II running. The search is performed for events with $P_{T}^{\tau}>10 \mathrm{GeV}$ and $20<\theta_{\tau}<120$. The distribution of events found is shown in Fig. 4, no excess over the SM predictions is observed in either $e^{+} p$ or $e^{-} p$ collisions. For events in the $P_{T}^{X}>25 \mathrm{GeV}$ region, 1 data event is observed compared to an expectation of $0.99 \pm 0.13$. It should be noted that the fraction of events arising from $W$ production in this search is significantly smaller than in the $e$ and $\mu$ searches.

\section{Summary}

The results of searches for events containing isolated leptons and large missing transverse momentum using data collected over the entire HERA running period have been presented. The latest electron- and muon-search results from $\mathrm{H} 1$ reveal no excess over the SM predictions for the $e^{-} p$ data, however the previously observed excess over SM predictions in $e^{+} p$ data persists. The ZEUS collaboration has also searched for isolated electrons and muons, no excess over SM predictions is observed. In the new $\tau$ search performed by the H1 collaboration no excess of events over the SM is observed. Collaboration between H1 and ZEUS has ensured that their most recent electron and muon searches are compatible, enabling combination at the event level. The results of the combined search for electrons and muons are compatible with the Standard Model.

\section{References}

[1] U. Baur, J. A. M. Vermaseren and D. Zeppenfeld, Nucl. Phys. B375, 3 (1992).

[2] K.-P. O. Diener, C. Schwanenberger and M. Spira, Eur. Phys. J. C25, 405 (2002), [hep-ph/0203269].

[3] H. Fritzsch and D. Holtmannspotter, Phys. Lett. B457, 186 (1999), [hep-ph/9901411].

[4] S. Y. Choi et al., arXiv:hep-ph/0612302.

[5] H1, C. Adloff et al., Eur. Phys. J. C5, 575 (1998), [hep-ex/9806009].

[6] H1, V. Andreev et al., Phys. Lett. B561, 241 (2003), [hep-ex/0301030].

[7] ZEUS, J. Breitweg et al., Phys. Lett. B471, 411 (2000), [hep-ex/9907023].

[8] ZEUS, S. Chekanov et al., Phys. Lett. B559, 153 (2003), [hep-ex/0302010].

[9] H1, A. Aktas et al., Eur. Phys. J. C48, 699 (2006), [hep-ex/0604022].

[10] ZEUS, S. Chekanov et al., Phys. Lett. B583, 41 (2004), [hep-ex/0311028].

[11] H1, E. Rizvi, These proceedings.

[12] H1, H1prelim-07-063:, Events with an isolated lepton (electron or muon) and missing transverse momentum at HERA. 
[13] ZEUS, ZEUS-prel-07-021:, Search for events with an isolated high-energy lepton and missing transverse momentum at HERA.

[14] H1, H1prelim-07-162:, Isolated leptons and missing pt: H1+zeus combination.

[15] H1, H1prelim-07-064:, Events with an isolated tau lepton and missing transverse momentum at HERA. 